The Chittagong Univ. J. Sci. 43(1): 31-40, 2021

\title{
Derivation of the Interfacial Surface Tension between Superconducting and Normal States within Ginzburg-Landau Theory
}

\author{
Neelufar Panna \\ Department of Physics, University of Chittagong, Chittagong-4331, Bangladesh \\ E-mail: neelcu@yahoo.com
}

Manuscript Submitted on 01/11/2020, Revised manuscript received on 24/01/2021, and accepted on $24 / 01 / 2021$

\begin{abstract}
An understanding of the front propagation in a dynamically unstable state requires a theory that treats the superconducting-normal ( $\mathrm{SN}$ ) interfaces as a free boundary problem. The relevance of an interfacial representation based on dynamics of the superconducting order parameter and vector potential. When magnetic flux diffuses into the normal phases the interfacial dynamics can be determined by the free boundary model. This model involves surface tension for the motion of the interface which has been calculated by the exact solution of TDGL equation [8].This analytical result of the surface tension agrees with that of Osborn and Dorsey [9].
\end{abstract}

Keywords: Front propagation in superconductor, Ginzburg-Landau equation, exact solution, surface tension.

DOI: https://doi.org/10.3329/cujs.v43i1.57332

বিশৃংখল গতীয় অবস্থায় অতিপরিবাহীতে আন্তঃপৃষ্ঠবিষ্তারণ এর জন্য একটি তত্ত্বের প্রয়োজন যেটি অতিপরিবাহীস্বাভাবিক অন্তঃপৃষ্ঠকে একটি মুক্ত-বাউন্ডারী সমস্যা হিসেবে বিবেচনা করে। অতিপরিবাহীতে অর্ডার প্যারামিটার এবং ভেক্টর পটেনশিয়াল এর গতি আন্তঃপৃষ্ঠবিস্তারণের উপর নির্ভরশীল। যখন চৌম্বকীয় ফ্লাক্স স্বাভাবিক দশায় প্রবেশ করে মুক্ত-বাউন্ডারী মডেল দ্বারা অতিপরিবাহীর আন্তঃপৃষ্ঠ গতিবিদ্যা নির্ণয় করা যায়। আন্তঃপৃষ্ঠ গতির জন্য পৃষ্ঠটান এ মডেলের অন্তর্ভূক্ত যা TDGL সমীকরনের এক্সাকট্ সমাধান ব্যবহার করেও নির্ণয় করা হয়েছে। পৃষ্ঠটানের এ অ্যানালাইটিকাল ফলাফল Osborn and Dorsey [9] এর ফলাফলের সাথে সংগতিপূর। । 


\section{Introduction}

The interfacial surface tension between normal $(\mathrm{N})$ and superconducting $(\mathrm{S})$ phases in a superconductor is a great attraction to different research groups for studying the dynamical properties of the interface. It has shown that when a type-I superconductor is subjected to a magnetic field quench, the magnetic flux expelled. As a result superconducting nucleus grows in the background normal-phase. After growing the superconducting phase the possibility arises for the surface tension of the SN (superconducting-normal) interface to be positive (type-I superconductor) [1], [2]. If the surface tension is negative, the interface breaks down and type-II superconductor results. The motion of the interface into the normal state leads to dynamical instabilities. From recent studies it has shown that there is a strong relation between the dynamics of SN interfaces in a magnetic field and the motion of solid-liquid interfaces during solidification. The common thing of this relationship is the diffusion of magnetic field in the normal state by analogy with the diffusion of latent heat in crystallization. This suggests that diffusional instability e.g. Mullins-Sekerka instability should occur during flux invasion [3]. Studies of the time dependent Ginzburg-Landau (TDGL) equation confirmed these instabilities. The free boundary model has derived from the TDGL equation.

As a superconducting nucleus grows, the expelling flux (Meissner effect) creates eddy current in the normal phase. In the normal region the interface dynamics is given by a diffusion equation for the magnetic field [4].

$\partial_{t} \underline{h}=D_{H} \nabla^{2} \underline{h}$

where, $D_{H}=\frac{1}{4 \pi \sigma^{(n)}}$ is the magnetic field diffusion constant, $\sigma^{(n)}$ is the normal state conductivity.

For the magnetic field at the SN interface (denoted by the subscript i) the continuity equation is

$(\underline{\nabla} \times \underline{h}) \times\left.\hat{n}\right|_{i}=-\mathrm{D}_{\mathrm{H}} v_{n} \underline{h}_{i}$ 
where, $\hat{n}$ is the unit normal at the interface directed towards the normal phase, $v_{n}$ is the normal velocity of the planar interface, as sketched in Fig. 1.

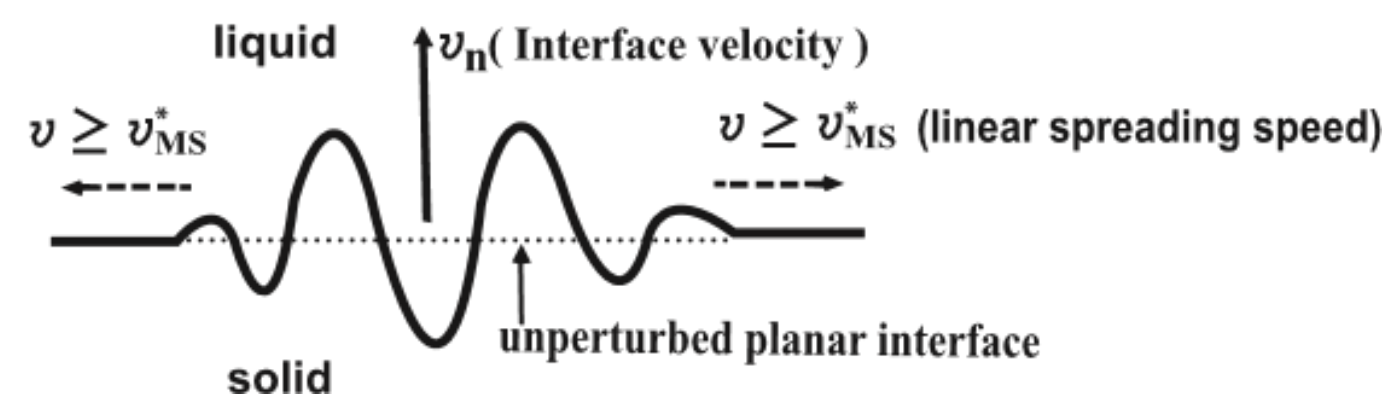

Figure 1. Mullins-Sekerka instability [3] along a growing solid-liquid interface. The unperturbed planar interface is growing in the vertical direction with a normal velocity $v_{n}$, shown by the dotted line. The dashed arrows indicate the propagation of the perturbation along the interface.

The field at the interface

$\underline{h}_{i}=H_{c}\left[1-\frac{4 \pi}{\mathrm{H}_{\mathrm{c}}{ }^{2}}\left(\sigma_{n s}+\beta^{-1} v_{n}\right)\right]$

Here, $H_{c}, \sigma_{n s}$ and $\beta$ are the critical field, surface tension, curvature and kinetic coefficient of the interface respectively.

In the conventional units the surface tension is defined in terms of dimensionless surface tension as

$\sigma_{n s}=\left(\frac{H_{c}^{2} \lambda}{4 \pi}\right) \bar{\sigma}_{n s}$

where, $\lambda$ is the penetration depth. 


\section{Neelufar Panna}

The aim of this paper is to obtain explicit formula for the surface tension of SN interface as a function of the GL parameter, $\kappa$. The paper is organized as follows. In section 2 surface tension has discussed and the exact solution of TDGL equation has used to derive surface tension. Section 3 provides the results of the calculations for surface tension of SN interface.

\section{Theory}

One of the important aspects of the dynamics of interface in superconductor is that there is a competition between dynamic instability which promotes the growth of highly ramified interface and surface tension, which favors a smooth interface.

In dimensionless units $[5,6,7]$ the one dimensional TDGL eq. in this case become

$$
\begin{aligned}
& \partial_{t} F=\frac{1}{\kappa^{2}} F^{\prime \prime}-Q^{2} F+F-F^{3} \\
& \bar{\sigma}_{t} Q=Q^{\prime \prime}-F^{2} Q
\end{aligned}
$$

where $F$ is the magnitude of the superconducting order parameter $\psi, Q$ is the gauge invariant vector potential related to the magnetic field as $h=\partial_{x} Q, \bar{\sigma}$ is the dimensionless normal state conductivity which is the ratio of the order parameter diffusion constant to the magnetic field diffusion constant.

In the previous paper [8] we made a minor contribution to the interface problem by considering the following generalized Ginzburg-Landau eq.:

$$
\begin{aligned}
& \left(1+\beta^{2}\right) F^{\prime \prime}+v F^{\prime}-Q^{2} F+a_{1} F+a_{2} F^{2}+a_{3} F^{3}=0 \\
& Q^{\prime \prime}+\sigma_{0} Q^{\prime}-F^{2} Q=0 \\
& \text { (taking } \left.\frac{1}{\kappa^{2}}=1+\beta^{2} \text { and } \bar{\sigma}=\sigma_{0}\right) \cdot a_{1}, a_{2} \text { and } a_{3} \text { are constant parameters. }
\end{aligned}
$$


The exact solutions of eqn.(2.3) and eq.(2.4) have constructed which shows a duality between order parameter and vector potential. The order parameter connects $F=1$ at $x=-\infty$ (the superconducting phase) to $F=0$ at $x=\infty$ (the normal phase).The solutions are

$$
\begin{aligned}
& F(x)=\frac{1}{1+\alpha e^{\xi x}} \\
& Q(x)=\frac{\alpha \beta e^{\xi x}}{1+\alpha e^{\xi x}}
\end{aligned}
$$

$\alpha$ is any arbitrary constant.

These solutions have used to calculate the surface tension of the SN interface.

We know the canonical form of the dimensionless surface tension of the SN interface is [4], [9]

$$
\bar{\sigma}_{n s}=\int_{-\infty}^{\infty}\left[-F^{2}+\frac{1}{2} F^{4}+\frac{1}{\kappa^{2}} F^{\prime 2}+Q^{2} F^{2}+\left(Q^{\prime}-\frac{1}{\sqrt{2}}\right)^{2}\right] d x
$$

Multiplying the equilibrium form of the 1 st G-L eq.(2.1) by $F^{\prime}$ and 2 nd G-L eq.(2.2) by $Q^{\prime}$ and then adding the two eqns. This result together with eq.(2.7) gives

$\bar{\sigma}_{n s}=2 \int_{-\infty}^{\infty}\left[\frac{1}{\kappa^{2}}\left(F^{\prime}\right)^{2}+\left(Q^{\prime}\right)^{2}-\frac{1}{\sqrt{2}} Q^{\prime}\right] d x$

By using the identity

$$
-F^{2}+\frac{1}{2} F^{4}+F^{2} Q^{2}=\frac{1}{\kappa^{2}}\left(F^{\prime}\right)^{2}+Q^{\prime 2}-\frac{1}{2}
$$


it can be shown from eq.(2.8) that

$$
2 \int_{-\infty}^{\infty} d x\left(F^{\prime}\right)^{2}=2 \kappa^{2} \int_{-\infty}^{\infty} d x\left[F^{2}-F^{4}-F^{2} Q^{2}\right]
$$

And

$$
2 \kappa^{2} \int_{-\infty}^{\infty} d x\left[\frac{1}{\sqrt{2}} Q^{\prime}-\left(Q^{\prime}\right)^{2}\right]=2 \kappa^{2} \int_{-\infty}^{\infty} d x F^{2} Q^{2}
$$

Substituting eqs. (2.9) and (2.10) into eq. (2.8), we have the expression for the surface tension of SN interface

$$
\bar{\sigma}_{n s}=2 \int_{-\infty}^{0} F^{2} d x-2 \int_{-\infty}^{0} F^{4} d x-4 \int_{-\infty}^{0} F^{2} Q^{2} d x
$$

To solve this integral eq. let us set $z=1+\alpha e^{\xi x}$.

Solving the 1 st term of R.H.S. of eq. (2.11) gives

$$
2 \int_{-\infty}^{0} F^{2} d x=\frac{2}{\xi}\left[\frac{1}{z}+\log (z-1)-\log z\right]_{-\infty}^{0}
$$

2nd term of R.H.S. of eq. (2.11) gives

$$
2 \int_{-\infty}^{0} F^{4} d x=\frac{2}{\xi}\left[\frac{2 z+1}{2 z^{2}}-\log \frac{z}{z-1}+\frac{1}{3 z^{3}}\right]_{-\infty}^{0}
$$

last term gives

$$
4 \int_{-\infty}^{0} F^{2} Q^{2} d x=\frac{4 \beta^{2}}{\xi}\left[\frac{1}{3 z^{3}}-\frac{1}{2 z^{2}}\right]_{-\infty}^{0}
$$

Substituting eqs. (2.12) - (2.14) into eq. (2.11) 


$$
\begin{aligned}
& \bar{\sigma}_{n s}=\frac{2}{\xi}\left[\frac{1}{z}+\log (z-1)-\log z\right]_{-\infty}^{0}-\frac{2}{\xi}\left[\frac{2 z+1}{2 z^{2}}-\log \frac{z}{z-1}+\frac{1}{3 z^{3}}\right]_{-\infty}^{0}-\frac{4 \beta^{2}}{\xi}\left[\frac{1}{3 z^{3}}-\frac{1}{2 z^{2}}\right]_{-\infty}^{0} \\
& =\frac{1}{\xi}\left[\frac{2}{3 z^{3}}-\frac{3}{z^{2}}-\frac{4}{3 z^{3} \kappa^{2}}+\frac{2}{z^{2} \kappa^{2}}\right]_{-\infty}^{0} \\
& \therefore \bar{\sigma}_{n s}=\frac{1}{\xi}\left[\frac{2}{3\left(1+\alpha e^{\xi x}\right)^{3}}-\frac{3}{\left(1+\alpha e^{\xi x}\right)^{2}}-\frac{4}{3 \kappa^{2}\left(1+\alpha e^{\xi x}\right)^{3}}+\frac{2}{\kappa^{2}\left(1+\alpha e^{\xi x}\right)^{2}}\right]_{-\infty}^{0}
\end{aligned}
$$

(after putting the value of $\mathrm{z}$ ).

\section{Results and Discussion}

This paper presents an analytical derivation of surface tension of superconductingnormal interface in terms of G-L parameter $\kappa$.

Now, setting $\alpha=0.1$ eq. (2.16) becomes

$$
\bar{\sigma}_{n s}=\frac{1.49}{\kappa^{2}}-0.82
$$

is the surface tension in terms of $\kappa$ for which a superconducting front is obtained.

The result agrees with the result $\bar{\sigma}_{n s}=0.388\left(\frac{1}{2 \kappa^{2}}-1\right)$ of Osborn and Dorsey [9]. 
Figure 2 represents the graph for the positive surface tension of the $\mathrm{SN}$ interface for different values of $\kappa$, ranging $0.5<\kappa<3$, according to the analytical expression eq. (2.17) shows that the superconductor is of type $-\mathrm{I}$.

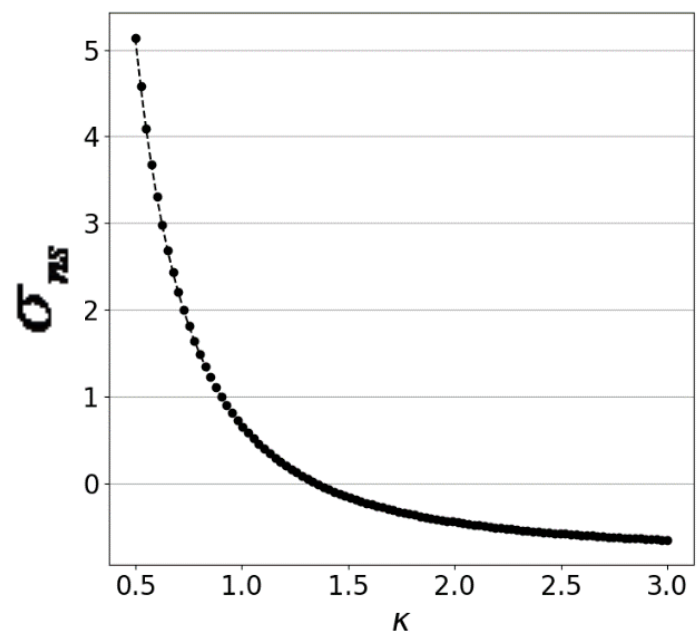

Figure 2. The dimensionless surface tension $\sigma_{n s}$ as a function of the GL parameter $\kappa$ for $0.5<\kappa<3$, calculated by eq. (2.17) 
Figure 3 shows the dependence of surface tension on $\kappa$ in the range $2<\kappa<10$. In this case the G-L parameter produce a negative contribution to the surface tension as a result of type- II superconductor.

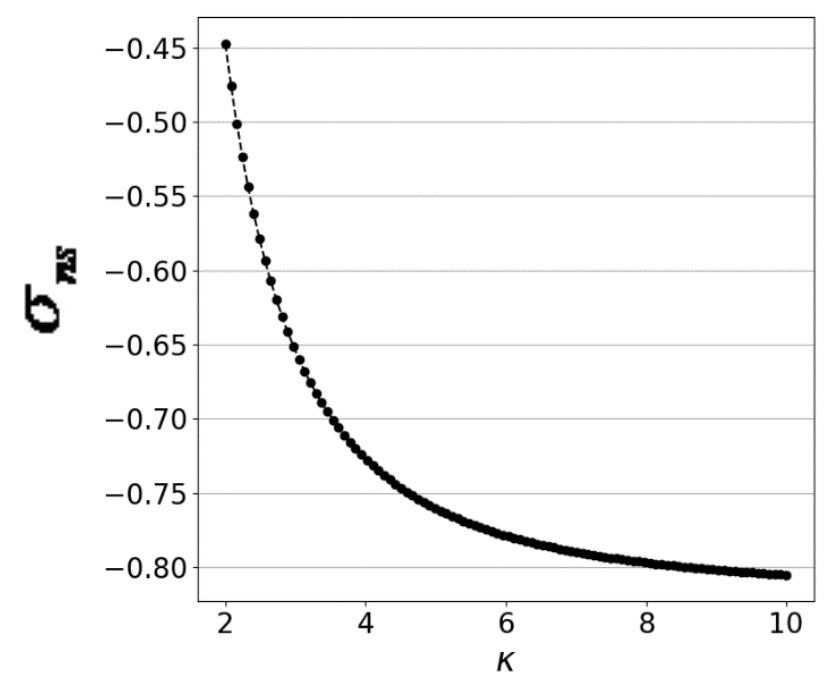

Figure 3. Dimensionless surface tension $\sigma_{n s}$ as a function of the GL parameter $\kappa$ for $2.0<\kappa<10.0$, calculated by eq. (2.17).

\section{Conclusion}

We know TDGL equation is widely used to calculate the surface tension of SN interface. The propagation of interface separating the superconducting and normal phases are produced after a quench to zero applied magnetic field. From a modified Gibbs-Thomson boundary condition for the magnetic field at the SN interface the dimensionless surface tension is calculated. It was known that the growth of superconducting phase into the normal phase should be dynamically unstable; such 
instabilities were discussed in the TDGL eq. of superconductivity for propagating interfaces. The dimensionless surface tension can be expressed in terms of the solutions of one dimensional TDGL eqs. Here the exact solutions of TDGL have used to calculate the surface tension. The result is in good agreement with the work of Osborn and Dorsey [9].

I would like to expand my work in two dimensional superconductivity such as TPS (Twinning plane superconductivity). It is possible to calculate the surface tension in the framework of GL theory.

\section{References}

[1] H. Frahm, S. Ullah and A. T. Dorsey, Phys. Rev. Lett. 1991, 66, 3067.

[2] F. Liu, M. Mondello and N. D. Goldenfeld, Phys. Rev. Lett. 1991, 66, 3071.

[3] W. W. Mullins and R. F. Sekerka, J. Appl. Phys. 1964, 35, 444.

[4] A.T. Dorsey, Ann.phys.1994, 233, 248.

[5] R. A. Fisher, Ann. Eugen., 1937, 7, 355.

[6] A. N. Kolmogorov, I. G. Petrovsii and N. S. Piskunov: Bull. Univ. Moscow, Ser. Int. A, 1937, 1, 1 .

[7] S. J. Di Bartolo and A. T. Dorsey, Phys. Rev. Lett., 1996, 77(21), 4442.

[8] Neelufar Panna and J. N. Islam: Pramana-Indian Academy of Sciences J. Phys., 2013, 80(5), 895.

[9] J. C. Osborn and A.T. Dorsey,Phys Rev. B, 1994, 50(21), 15961.

The Chittagong Univ. J. Sc. Vol. 43(1), 2021 